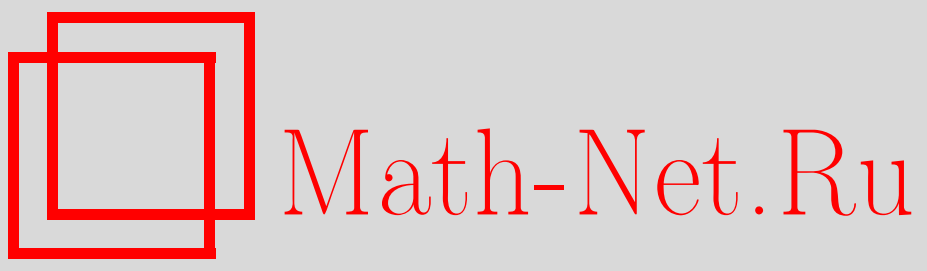

М. Л. Горбачук, О порядке роста операторной экспоненты на целых векторах, Функи. анализ и его прил., 2002, том 36, выпуск 1, 75-78

DOI: https://doi.org/10.4213/faa179

Использование Общероссийского математического портала MathNet.Ru подразумевает, что вы прочитали и согласны с пользовательским соглашением

http ://www . mathnet.ru/rus/agreement

Параметры загрузки :

IP : 54.224 .135 .184

26 апреля 2023 г., 09:29:57

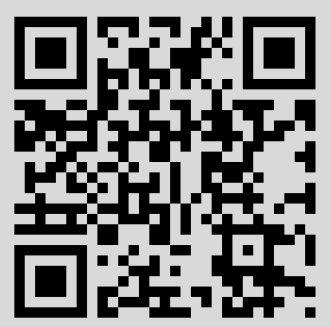




\title{
О порядке роста операторной экспоненты на целых векторах
}

\author{
(C) 2002. М. Л. ГОРБАЧУК
}

Пусть $A-$ замкнутый оператор в банаховом пространстве $X$ с нормой $\|\cdot\|$. Вектор $x \in C^{\infty}(A)=\bigcap_{n \in \mathbb{N}_{0}=\{0,1,2, \ldots\}} \mathscr{D}\left(A^{n}\right)(\mathscr{D}(\cdot)$ - область определения оператора) называется целым вектором оператора $A$, если ряд

$$
e^{\lambda A} x=\sum_{n=0}^{\infty} \frac{\lambda^{n} A^{n} x}{n !}
$$

сходится для всех $\lambda \in \mathbb{C}$. Ясно, что вектор $x \in C^{\infty}(A)$ является целым для оператора $A$ тогда и только тогда, когда для каждого положительного числа $\alpha$ существует постоянная $c=c(\alpha)>0$, такая, что $\left\|A^{n} x\right\| \leqslant c \alpha^{n} n^{n}$.

Если оператор $A$ ограничен, то любой вектор $x \in X$ является целым вектором этого оператора, а $e^{\lambda A} x-$ целой вектор-функцией экспоненциального типа, т. е. ее порядок не превышает 1. Напомним, что по определению целая векторфункция $y(\lambda)$ имеет конечный порядок, если существует такое число $k>0$, что $\|y(\lambda)\| \leqslant \exp \left(|\lambda|^{k}\right)$ для достаточно больших $|\lambda|$. Точная нижняя грань таких $k$ называется порядком этой вектор-функции и в дальнейшем обозначается через $\rho(y)$. Под типом целой вектор-функции $y(\lambda)$ порядка $\rho=\rho(y)$ понимается число $\sigma(y)=\inf \left\{a>0:\|y(\lambda)\| \leqslant \exp \left(a|\lambda|^{\rho}\right)\right\}$. При $\sigma(y)=0$ целая векторфункция $y(\lambda)$ имеет так называемый минимальный, а при $0<\sigma(y)<\infty-$ нормальный тип.

Если пространство $X$ гильбертово, а $A$ - ограниченный нормальный оператор в нем, то $\rho\left(e^{\lambda A} x\right)=1, \sigma\left(e^{\lambda A} x\right) \leqslant\|A\|$ и равенство $\sigma\left(e^{\lambda A} x\right)=0$ эквивалентно тому, что $A x=0$. Однако нетрудно привести пример ограниченного оператора, не являющегося нормальным и такого, что, какими бы ни были $\rho \in(0,1]$ и $\sigma \in[0, \infty)$, найдется вектор $x \in X$, для которого $\rho\left(e^{\lambda A} x\right)=\rho, \sigma\left(e^{\lambda A} x\right)=\sigma$.

Неограниченный оператор в банаховом пространстве может вообще не иметь целых векторов, отличных от нулевого, а если таковые $x$ и существуют, то порядок и тип вектор-функций $e^{\lambda A} x$ для таких операторов $A$ могут быть какими угодно. Описать их зависимость от степени гладкости элемента $x$ относительно оператора $A$ - цель этой заметки. При $\rho\left(e^{\lambda A} x\right) \leqslant 1,0 \leqslant \sigma\left(e^{\lambda A} x\right)<\infty$ такая зависимость была изучена в [1]. Поведение $\rho\left(e^{\lambda A} x\right)$ и $\sigma\left(e^{\lambda A} x\right)$ в зависимости от порядка наилучшего приближения вектора $x$ целыми векторами экспоненциального типа оператора $A$ (их определение см. ниже) и при дополнительных условиях на резольвенту оператора $A$ исследовано в $[2,3]$. Правда, содержащиеся в [3] теоремы 1.2 и 1.4 сформулированы некорректно, так как введенная там функция $\sigma_{E}(f, A)$ может принимать лишь два значения, а именно 0 и $\infty$.

Итак, пусть $A-$ замкнутый линейный оператор в банаховом пространстве $X$ и $x$ - его целый вектор. Будем говорить, что $x$ имеет конечный порядок, если существует число $\beta \in(-\infty, 1)$, такое, что для достаточно больших $n\left(n>n_{0}(\beta)\right)$

$$
\left\|A^{n} x\right\| \leqslant n^{n \beta} .
$$


Точную нижнюю грань таких $\beta$ назовем порядком вектора $x$ и обозначим через $\widetilde{\rho}(x)$. Очевидно, что

$$
\widetilde{\rho}(x)=\varlimsup_{n \rightarrow \infty} \frac{\ln \left\|A^{n} x\right\|}{n \ln n} .
$$

Определим тип $\widetilde{\sigma}(x)$ целого вектора $x$ порядка $\widetilde{\rho}(x)$ формулой

$$
\widetilde{\sigma}(x)=\inf \left\{\alpha>0:\left\|A^{n} x\right\| \leqslant \alpha^{n} n^{n \widetilde{\rho}(x)}, n>n_{0}(\alpha)\right\} .
$$

Нетрудно видеть, что

$$
\widetilde{\sigma}(x)=\varlimsup_{n \rightarrow \infty} \frac{\sqrt[n]{\left\|A^{n} x\right\|}}{n^{\widetilde{\rho}(x)}} .
$$

Если $\widetilde{\sigma}(x)=0$, то будем говорить, что $x-$ вектор минимального типа, а если $0<\widetilde{\sigma}(x)<\infty-$ нормального типа.

Положим

$$
\mathfrak{G}_{\{\beta\}}(A)=\bigcup_{\alpha>0} \mathfrak{G}_{\beta}^{\alpha}(A), \quad \mathfrak{G}_{(\beta)}(A)=\bigcap_{\alpha>0} \mathfrak{G}_{\beta}^{\alpha}(A),
$$

где для чисел $\alpha>0$ и $\beta \in(-\infty, 1)$

$$
\mathfrak{G}_{\beta}^{\alpha}(A)=\left\{x \in C^{\infty}(A) \mid \exists c=c(x)>0 \forall n \in \mathbb{N}_{0}:\left\|A^{n} x\right\| \leqslant c \alpha^{n} n^{n \beta}\right\} .
$$

Элементы пространства $\mathfrak{G}_{\{0\}}(A)$ называются целыми векторами экспоненциального типа оператора $A$ (см. [4]). Тот факт, что целый вектор $x$ оператора $A$ имеет конечный порядок $\widetilde{\rho}=\widetilde{\rho}(x)$ и нормальный (минимальный) тип, равнозначен включению $x \in \mathfrak{G}_{\{\beta\}}(A)\left(x \in \mathfrak{G}_{(\beta)}(A)\right)$ с $\beta=\widetilde{\rho}$.

Теорема 1. Пусть $x$ - целый вектор оператора $А$. Для того чтобы иелая вектор-функция $e^{\lambda A} x$ имела конечный порядок $\rho$, необходимо и достаточно, чтобы порядок $\widetilde{\rho}$ вектора $x$ был конечен. Ее тип $\sigma$ является нормальным (минимальнымм тогда и только тогда, когда нормален (минимален) тип $\widetilde{\sigma}$ вектора $x$. При этом между $\rho$ и $\widetilde{\rho}, \sigma$ и $\widetilde{\sigma}$ имеют место соотношения

$$
\tilde{\rho}=1-1 / \rho, \quad \widetilde{\sigma}=\left(e^{1-\rho} \sigma \rho\right)^{1 / \rho} .
$$

ДокАЗАТЕЛЬСтво. Предположим, что вектор-функция $y(\lambda)=e^{\lambda A} x$ имеет конечный порядок $\rho(y)=\rho$ и тип $\sigma(y)=\sigma$. Тогда для малых $\varepsilon>0$

$$
\|y(\lambda)\| \leqslant c \exp \left((\sigma+\varepsilon)|\lambda|^{\rho}\right), \quad c=\text { const . }
$$

Поскольку при $n \in \mathbb{N}_{0}$

то

$$
\frac{d^{n} y(\lambda)}{d \lambda^{n}}=A^{n} y(\lambda)
$$

$$
\left\|A^{n} x\right\|=\left\|A^{n} y(0)\right\|=\left\|y^{(n)}(0)\right\|=\frac{n !}{2 \pi}\left\|\int_{|\lambda|=r} \frac{y(\lambda)}{\lambda^{n+1}} d \lambda\right\| \leqslant c n ! \frac{e^{(\sigma+\varepsilon) r^{\rho}}}{r^{n}},
$$

где $r>0$ любое. Учитывая, что минимум функции $e^{\sigma r^{\rho}} / r^{n}$ достигается в точке $r=(n /(\sigma \rho))^{1 / \rho}$, с помощью формулы Стирлинга получаем

$$
\left\|A^{n} x\right\|<c_{1}\left(\left(e^{1-\rho} \sigma \rho\right)^{1 / \rho}+\varepsilon_{1}\right)^{n} n^{n(\rho-1) / \rho} .
$$

Здесь $\varepsilon_{1}=\varepsilon_{1}(\varepsilon) \rightarrow 0$ при $\varepsilon \rightarrow 0,0<c_{1}=$ const. Таким образом,

$$
\tilde{\rho} \leqslant(\rho-1) / \rho, \quad \tilde{\sigma} \leqslant\left(e^{1-\rho} \sigma \rho\right)^{1 / \rho} .
$$


Обратно, пусть порядок $\widetilde{\rho}$ вектора $x$ и его тип $\widetilde{\sigma}$ конечны. Из равенства

$$
\rho(y)=\varlimsup_{n \rightarrow \infty} \frac{n \ln n}{\ln \left(n ! /\left\|A^{n} x\right\|\right)}
$$

(см. [5, с. 13, теорема 2]) и формулы Стирлинга следует соотношение

$$
\rho \leqslant \varlimsup_{n \rightarrow \infty} \frac{n \ln n}{\ln \left(n ! /\left(c \widetilde{\sigma}^{n} n^{n \widetilde{\rho}}\right)\right)}=\frac{1}{1-\widetilde{\rho}} .
$$

Первое из неравенств в (1) и соотношение (2) приводят к выводу, что $\rho=$ $1 /(1-\widetilde{\rho})$.

Для нахождения типа $\sigma$ воспользуемся формулой

$$
(e \sigma(y) \rho)^{1 / \rho}=\varlimsup_{n \rightarrow \infty}\left(n^{1 / \rho} \sqrt[n]{\frac{\left\|A^{n} x\right\|}{n !}}\right)
$$

(см. [5, с. 13, теорема 2]). Тогда

$$
(e \sigma \rho)^{1 / \rho} \leqslant \varlimsup_{n \rightarrow \infty} n^{1 / \rho} \sqrt[n]{\frac{(\widetilde{\sigma}+\varepsilon)^{n} n^{n \tilde{\rho}}}{n !}}=e(\widetilde{\sigma}+\varepsilon) .
$$

Так как $\varepsilon>0$ произвольно, то

$$
(e \sigma \rho)^{1 / \rho} \leqslant e \widetilde{\sigma}
$$

Второе неравенство в (1) и неравенство (3) дают $\widetilde{\sigma}=\left(e^{1-\rho} \sigma \rho\right)^{1 / \rho}$. Теорема доказана.

СлЕДСТВИЕ 1. Для того итобы вектор-функц̧ия $y(\lambda)=e^{\lambda A} x$ была изелой вектор-функцией экспоненциального типа, необходимо и достаточно, чтобы $x$ был цзельм вектором экспоненциильного типа оператора А. При этом $\sigma(y)=\widetilde{\sigma}(x)$.

Следуя [6], для произвольного вектора $x \in X$, положим

$$
\mathscr{E}_{r}(x)=\inf _{g \in \mathfrak{G}_{\{0\}}(A): \widetilde{\sigma}(g) \leqslant r}\|x-g\| .
$$

Функция $\mathscr{E}_{r}(x)$ монотонно невозрастающая, и

$$
\left(\mathscr{E}_{r}(x) \rightarrow 0 \text { при } r \rightarrow \infty\right) \Longleftrightarrow\left(\overline{\mathfrak{G}_{\{0\}}(A)}=X\right) .
$$

В предположении замкнутости пространства $\mathfrak{G}_{0}^{\alpha}(A)$ в $X$ множество $\bigcap_{\varepsilon>0} \mathfrak{G}_{0}^{\alpha+\varepsilon}(A)$ также замкнуто в $X$. Из равносильности соотношений $x \in \bigcap_{\varepsilon>0} \mathfrak{G}_{0}^{r+\varepsilon}(A)$ и $\widetilde{\sigma}(x) \leqslant r$ вытекает, что

$$
\left(x \in \bigcap_{\varepsilon>0} \mathfrak{G}_{0}^{r_{0}+\varepsilon}(A)\right) \Longleftrightarrow\left(\mathscr{E}_{r}(x)=0 \text { при } r>r_{0}\right) .
$$

В этом случае следствие 1 можно переписать в такой эквивалентной форме: вектор-функция $y(\lambda)=e^{\lambda A} x$, где $x$ - целый вектор оператора $A$, имеет порядок $\rho \leqslant 1$ и конечный тип тогда и только тогда, когда существует число $r_{0} \geqslant 0$, такое, что $\mathscr{E}_{r}(x)=0$ при $r>r_{0}$; если для некоторого $r_{0}>0$ при $0<r<r_{0}$ имеет место неравенство $\mathscr{E}_{r}(x)>0$, а при $r>r_{0}-$ равенство $\mathscr{E}_{r}(x)=0$, то $x$ - целый вектор экспоненциального типа оператора $A$, а вектор-функция $y(\lambda)=e^{\lambda A} x$ имеет первый порядок роста и нормальный тип. Это утверждение при дополнительном условии на поведение резольвенты замкнутого оператора $A$ было установлено в 
[2, с. 118, теорема 5.3]. Следует подчеркнуть, что нами никаких ограничений на оператор $A$, кроме замкнутости пространства $\mathfrak{G}_{0}^{\alpha}(A)$ в $X$, не налагается. Заметим также, что из теоремы 1 при условии на оператор $A$, обеспечивающем возможность описания некоторых подпространств из $C^{\infty}(A)$ в терминах поведения функции $\mathscr{E}_{r}(x)$, как это сделано в $[6]$, а затем в $[2,3]$, можно получить теорему 5.4 из [2] или, что то же самое, теорему 1.3 из [3].

\section{ЛИТЕРАТУРА}

1. Горбачук М. Л. Математические методы и физико-механические поля, 41, 7-12 (1998). 2. Радзиевский Г. В. Матем. сб., 189, вып. 4, 83-124 (1999). 3. Радзиевский Г. В. Функц. анализ и его прил., 33, вып. 2, $43-57$ (1999). 4. Радыно Я. В. Докл. АН БССР, 27, № 9, 791-793 (1983). 5. Левин Б. Я. Распределение корней целых функций. ГИТТЛ, М., 1956. 6. Горбачук В. У., Горбачук М. Л. Укр. мат. журн., 48, № 4, 616-628 (1995).

Институт математики НАН Украины

email: imath@horbach.kiev.ua

Поступило в редакцию 11 января 2000 г.

УДК 515.16

\section{Комбинаторика мультикраевых особенностей серии $B_{n}^{l}$ и числа Бернулли-Эйлера*}

(C) 2002. О. Н. КАРПЕНКОВ

Введение. В работах $[1,2]$ В. И. Арнольд, в частности, установил, что компоненты множества вполне правильных М-морсификаций краевых особенностей серии $B_{n}$ связаны с комбинаторикой соответствующих конусов Спрингера, а их количество равно числу Бернулли-Эйлера $K_{n}$.

В этой заметке рассматриваются обобщения краевых особенностей $B_{n}$ функций на прямой для случая, когда край состоит из конечного числа $(l)$ точек. Такие особенности $B_{n}^{l}$ возникают и в больших размерностях, когда край - иммерсированная гиперповерхность.

Получено рекуррентное соотношение между количеством связных компонент множества вполне правильных $M$-морсификаций для различных значений $n$ и $l$.

Автор выражает благодарность В. И. Арнольду, В. М. Закалюкину и С. К. Ландо за оказанную помощь.

\section{Основные определения и понятия.}

ОПРеДЕлЕниЕ 1. Вполне правильная М-морсификация мультикраевой особенности $B_{n}^{l}$ - это многочлен, все критические точки которого вещественны и различны, причем значения во всех критических точках, а также и значения во всех краевых точках $x=b_{i}$ различны.

Заметим, что краевые точки у нас пронумерованы, иначе нужно было бы рассматривать фактор пространства $\mathbb{R}^{k}$ по группе перестановок координат. Краевые точки должны быть пронумерованы, так как они соответствуют разным прообразам, которые не переставляются.

*Работа поддержана грантами РФФИ-00-15-96084 и РФФИ-01-01-00803. 